\title{
Ultrafast and High-Yield Polaronic Exciton Dissociation in Two-Dimensional Perovskites
}

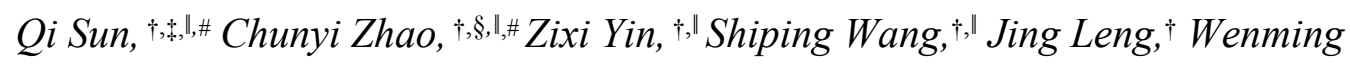
Tian, ${ }^{*}$, and Shengye $\mathrm{Jin}^{*}, \dagger$

†State key laboratory of molecular reaction dynamics and the dynamic research center for energy and environmental materials, Dalian Institute of Chemical Physics, Chinese Academy of Sciences, Dalian 116023, China

¥MIT Key Laboratory of Advanced Display Materials and Devices, Institute of Optoelectronics \& Nanomaterials, College of Materials Science and Engineering, Nanjing University of Science and Technology, Nanjing 210094, China

${ }^{\S}$ Anhui Province Key Laboratory of Optoelectronic Material Science and Technology, School of Physics and Electronic Information, Anhui Normal University, Wuhu, 241002, China.

"University of Chinese Academy of Sciences, Beijing 100049, China 


\section{Experimental Section}

\section{Synthesis of the $(\mathrm{PEA})_{2}(\mathrm{MA})_{\mathrm{n}-1} \mathrm{~Pb}_{\mathrm{n}} \mathrm{I}_{3 \mathrm{n}+1} 2 \mathrm{D}$ perovskites}

All chemicals are of analytical grade and directly used without further purification.

$(\mathbf{P E A})_{2} \mathbf{P b I}_{4}(\boldsymbol{n}=\mathbf{1}): \mathrm{PbI}_{2}(0.1 \mathrm{mmol})$ and PEAI $(0.1 \mathrm{mmol})$ were dissolved in 4 $\mathrm{mL}$ of $\mathrm{HI}$ solution $\left(57 \mathrm{wt} \%\right.$ in $\left.\mathrm{H}_{2} \mathrm{O}\right)$ with stirring and heating to $130{ }^{\circ} \mathrm{C}$. The plate-like single crystals were obtained after the solution was cooled down to room temperature $\left(\sim 20^{\circ} \mathrm{C} / \mathrm{h}\right)$. Then the single crystals were washed with diethyl ether and dried at $60{ }^{\circ} \mathrm{C}$ under vacuum for $24 \mathrm{~h}$ before exfoliation.

$(\mathbf{P E A})_{2} \mathbf{M A P b}_{2} \mathbf{I}_{7}(\boldsymbol{n}=\mathbf{2}): \mathrm{PbI}_{2}(0.4 \mathrm{mmol}), \mathrm{PEAI}(0.17 \mathrm{mmol})$ and MAI $(0.67$ mmol) were dissolved in $2 \mathrm{~mL}$ of $\mathrm{HI}$ solution $\left(57 \mathrm{wt} \%\right.$ in $\mathrm{H}_{2} \mathrm{O}$ ) with stirring and heating to $130{ }^{\circ} \mathrm{C}$. The following steps were carried out in a similar way to $n=1$ samples.

$(\mathbf{P E A})_{2}(\mathbf{M A})_{2} \mathbf{P b}_{3} \mathbf{I}_{10}(\boldsymbol{n}=\mathbf{3}): \mathrm{PbI}_{2}(0.4 \mathrm{mmol})$, PEAI $(0.067 \mathrm{mmol})$ and MAI ( $0.67 \mathrm{mmol})$ were dissolved in $2 \mathrm{~mL}$ of $\mathrm{HI}$ solution $\left(57 \mathrm{wt} \%\right.$ in $\left.\mathrm{H}_{2} \mathrm{O}\right)$ with stirring and heating to $130{ }^{\circ} \mathrm{C}$. The following steps were carried out in a similar way to $n=1$ samples.

$(\mathbf{P E A})_{\mathbf{2}}(\mathbf{M A})_{\mathbf{3}} \mathbf{P b}_{\mathbf{4}} \mathbf{I}_{\mathbf{1 3}}(\boldsymbol{n}=\mathbf{4}): \mathrm{PbI}_{2}(0.4 \mathrm{mmol}), \mathrm{PEAI}(0.03 \mathrm{mmol})$ and MAI (0.67 mmol) were dissolved in $3 \mathrm{~mL}$ of $\mathrm{HI}$ solution $\left(57 \mathrm{wt} \%\right.$ in $\mathrm{H}_{2} \mathrm{O}$ ) with stirring and heating to $130{ }^{\circ} \mathrm{C}$. The following steps were carried out in a similar way to $n=1$ samples. 


\section{Ultrafast Transient Absorption Spectroscopy}

The femtosecond transient absorption setup is based on a regenerative amplified Ti:sapphire laser system from Coherent $(800 \mathrm{~nm}, 35 \mathrm{fs}, 6 \mathrm{~mJ} /$ pulse, and $1 \mathrm{kHz}$ repetition rate), nonlinear frequency mixing techniques and the Famto-TA100 spectrometer (Time-Tech Spectra). Briefly, the $800 \mathrm{~nm}$ output pulse from the regenerative amplifier was split in two parts with a 50\% beam splitter. The transmitted part was used to pump a TOPAS Optical Parametric Amplifier (OPA) which generates a wavelength-tunable laser pulse from $250 \mathrm{~nm}$ to $2.5 \mu \mathrm{m}$ as pump beam. The reflected $800 \mathrm{~nm}$ beam was split again into two parts. One part with less than $10 \%$ was attenuated with a neutral density filter and focused into a $2 \mathrm{~mm}$ thick sapphire window to generate a white light continuum (WLC) from $420 \mathrm{~nm}$ to $800 \mathrm{~nm}$ used for probe beam. The probe beam was focused with an Al parabolic reflector onto the sample. After the sample, the probe beam was collimated and then focused into a fiber-coupled spectrometer with CMOS sensors and detected at a frequency of $1 \mathrm{kHz}$. The intensity of the pump pulse used in the experiment was controlled by a variable neutral-density filter wheel. The delay between the pump and probe pulses was controlled by a motorized delay stage. The pump pulses were chopped by a synchronized chopper at $500 \mathrm{~Hz}$ and the absorbance change was calculated with two adjacent probe pulses (pump-blocked and pump-unblocked). We prepared the samples by exfoliating repeatedly with a scotch tape until the sample became thin flakes on the tape. Then the flakes were transferred to a glass slip for measurements. 


\section{Femtosecond PL Up-conversion Spectroscopy}

The excitation source was a Pharos laser $(1030 \mathrm{~nm}, 100 \mathrm{kHz}, 230 \mathrm{fs}$ pulse-duration; Light Conversion), which was used to pump a TOPAS OPA to generate wavelength-tunable excitation pulses and the fundamental radiation (1030 $\mathrm{nm})$ of the laser system is typically used as the gate beam. The PL from samples is mixed with the gate beam into a $\mathrm{BBO}\left(\beta-\mathrm{BaB}_{2} \mathrm{O}_{4}\right)$ of type II crystal to produce sum-frequency signal. The delay between the PL and gate beam was controlled by a motorized delay stage. The sum-frequency signals were measured by a PMT detector. ${ }^{1}$ We prepared the samples by exfoliating repeatedly with a scotch tape until the sample became thin flakes on the tape. Then the flakes were transferred to a glass slip for measurements.

\section{Time-Resolved Confocal PL Measurement}

The PL imaging measurements were performed on the laser-scanned confocal imaging microscopy coupled with time-correlated single photon counting (TCSPC). Excitation of the sample was achieved with a $405 \mathrm{~nm}$ pulsed laser (PIXEA-405, Aurea Technology, France) with $\sim 35$ ps pulse width that is focused by objective lens $(100 \times, N A=0.95)$ to a near diffraction-limited spot. The laser intensity at samples was adjusted by a neutral density filter and measured with a power meter (PM100D S130VC, Thorlabs, USA). Each scanning contains $256 \times 256$ pixels. The fluorescence signal was collected using two high speed detectors (HPM-100-50 and HPM-100-40, Hamamatsu, Japan). We selected appropriate optical filters for perovskite and "edge 
state" PL collection of different 2D perovskite crystals with 565-625 nm, 600-640 $\mathrm{nm}, 630-670 \mathrm{~nm}$ for $\mathrm{n}=2,3,4$ perovskites excitonic emission and $>710 \mathrm{~nm}$ for edge state emission. Besides, a pinhole $(d<1 \mathrm{~mm})$ was placed before the detector to ensure that only PL from a diffraction-limited spot was observed.

\section{Steady-State Spectroscopy}

To obtain steady state PL emission spectra, a setup which mainly consists of a monochromator (SpectraPro-HRS-300, Princeton Instruments, USA) and a charge coupled device (CCD) camera (PIXIS 100, Princeton Instruments, USA) was used, and the samples were excited by a $405 \mathrm{~nm}$ pulsed laser (35 ps pulse width, PIXEA-405, Aurea Technology, France). The steady state absorption spectra were recorded by a UV-vis absorption spectrometer (Cary60, Agilent Technologies, USA).

\section{Temperature-Dependent Spectroscopic Measurements}

The temperature-dependent spectroscopic measurements were achieved by a temperature-controlled vacuum chamber (LNC-W, Lanhai Instrument) coupled with TA and PL-UC spectrometers.

\section{Structure Characterization}

SEM images were captured on a field emission scanning electron microscope (JSM-7800F, JEOL, Japan). X-ray diffraction (XRD) patterns were obtained on a X'pert Pro X-Ray Diffractometer (PANAlytical, Netherlands) using $\mathrm{Cu}$ Ka radiation. 


\section{Supplementary Note 1}

For the reversible exciton dissociation reaction, increasing the total carrier density (excitation intensity) will shift the exciton/free-carrier equilibrium toward generation of excitons, resulting in a decrease in dissociation efficiency $\left(\eta_{\mathrm{dis}}\right)$. With close-bandgap excitation ( $\left.E_{\text {exc. }} \approx E_{\mathrm{bg}}\right)$, we assume all of carriers are generated in the form of exciton at initial time $(t \approx 0 \mathrm{ps}$, Figure 3 and S15). After achieving equilibrium, the densities of exciton and free-carrier can be described as:

$$
\begin{gathered}
{[X]=\left(1-\eta_{\text {dis }}\right)[T C]} \\
{[F C]=\eta_{\text {dis }}[T C]}
\end{gathered}
$$

where the $[\mathrm{TC}]$ is the density of total carriers, and $[\mathrm{FC}]=[\mathrm{e}]=[\mathrm{h}]$. According to equilibrium constant $K=[\mathrm{FC}]^{2} /[\mathrm{X}]$, the $\eta_{\text {dis }}$ can be described as a function of $K$ and [TC]:

$$
\eta_{\text {dis }}=\frac{-K+\sqrt{K^{2}+4 K[T C]}}{2[T C]}
$$

Therefore, increasing the density of total carriers (or excitation intensity) leads to a decreased $\eta_{\text {dis }}$, as reflected by the smaller amplitude of fast decay in PL-UC kinetics.

\section{Supplementary Note 2}

The PL intensity $\left(I_{\mathrm{PL}}\right)$ of $2 \mathrm{D}$ perovskites can be determined by the radiative recombination of exciton and free-carrier:

$$
I_{P L} \propto k_{r}[X]+k_{r}^{\prime}[F C]^{2}
$$

where the $[\mathrm{X}]$ and $[\mathrm{FC}]$ are density of exciton and free-carrier, and the $k_{\mathrm{r}}$ and $k_{\mathrm{r}}$ are their radiative rate constants, respectively. Our results demonstrate that the major luminescent carrier specie is exciton in 2D perovskites (see Figure S9 and S10) and 
we therefore ignore the contribution of free-carrier to PL, i.e., $I_{\mathrm{PL}} \propto[\mathrm{X}]$. The kinetics equation of exciton can be described as:

$$
\frac{d[X]}{d t}=-k_{r}[X]-k_{n r}[X]-k_{d i s}[X]+k_{r b}[F C]^{2}
$$

where the $k_{\mathrm{nr}}$ and $k_{\mathrm{dis}}$ are the nonradiative and dissociation rate constants of exciton, respectively; $k_{\mathrm{rb}}$ is the rate constant of free-carriers rebound into excitons. Because the initial density of total carrier $[\mathrm{TC}]_{0}$ is fixed by excitation flux, which is proportional to excitation power $(P)$. Thus, Eq. S5 can be rewritten as a function of $[\mathrm{TC}]_{0}$ and initial dissociation efficiency $\left(\eta_{\text {dis }, 0}\right)$ :

$$
\begin{gathered}
\frac{d[X]}{d t}=-\left(k_{r}+k_{n r}+k_{d i s}\right)\left(1-\eta_{d i s, 0}\right)[T C]+k_{r b} \eta_{d i s, 0}^{2}[T C]^{2} \\
{[T C]_{0} \propto P}
\end{gathered}
$$

Therefore, the constant $\alpha$ in the law of $I_{0}(P) \propto P^{\alpha}$ reflects the initial distribution of carrier types: i) if all of photogenerated carriers are in the form of exciton, i.e., $\eta_{\mathrm{dis}, 0}=$ $0, \mathrm{~d}[\mathrm{X}] / \mathrm{d} t$ is proportional to the first power of $P$, therefore $\alpha=1$; ii) if the carriers totally generated as free-carriers, $\eta_{\mathrm{dis}, 0}=1$, the $\alpha$ equals to 2 due to the $\mathrm{d}[X] / \mathrm{d} t \propto P^{2}$; iii) and for the case of coexistence of both exciton and free-carrier, yielding $1<\alpha<2$.

\section{Supplementary Note 3}

The photoluminescence quantum yield (PLQY) is related to the ratio of radiative recombination rate to the total carrier depopulation rate, where the kinetics equation of total carrier decay can be obtained by the sum of depopulation rates of exciton and free-carrier:

$$
\begin{gathered}
\frac{d[X]}{d t}=-k_{r}[X]-k_{n r}[X]-k_{d i s}[X]+k_{r b}[F C]^{2} \\
\frac{d[F C]}{d t}=-k_{n r}^{\prime}[F C]+k_{d i s}[X]-k_{r b}[F C]^{2}
\end{gathered}
$$




$$
\frac{d[T C]}{d t}=\frac{d[X]}{d t}+\frac{d[F C]}{d t}=-k_{r}[X]-k_{n r}[X]-k_{n r}^{\prime}[F C]
$$

where the $k_{\mathrm{nr}}^{\prime}$ is nonradiative recombination rate constant of free-carriers. Under the equilibrium condition:

$$
\begin{gathered}
k_{d i s}[X]=k_{r b}[F C]^{2} \\
{[F C]=\frac{k_{d i s}^{1 / 2}}{k_{r b}^{1 / 2}}[X]^{1 / 2}=K^{1 / 2}[X]^{1 / 2}}
\end{gathered}
$$

where the $K$ is equilibrium constant, $K=k_{\mathrm{dis}} / k_{\mathrm{rb}}$. Therefore, the kinetics equation of total carrier decay (Eq. S9) can be described as:

$$
\frac{d[T C]}{d t}=-\left(k_{r}+k_{n r}\right)[X]-k_{n r}^{\prime} K^{1 / 2}[X]^{1 / 2}
$$

Therefore, the PLQY is determined by using the following equation:

$$
\mathrm{PLQY}=\frac{k_{r}[X]}{d[T C] / d t}=\frac{k_{r}}{\left(k_{r}+k_{n r}\right)+k_{n r}^{\prime} K^{1 / 2}[X]^{-1 / 2}}
$$

According to Eq. S13, if there is no additional nonradiative recombination channel for free-carrier (i.e., $k_{\mathrm{nr}}^{\prime}=0$ ), the exciton dissociation process should not affect the PLQY of 2D perovskites QWs.

\section{Supplementary Note 4}

The carrier density is estimated by using absorption coefficient $(\varepsilon)$ of $3.75 \times 10^{4}$ $\mathrm{cm}^{-1}$ (550 nm exc.) for $n=2,3.1 \times 10^{4} \mathrm{~cm}^{-1}$ for $n=3\left(600 \mathrm{~nm}\right.$ exc.) and $2.75 \times 10^{4}$ $\mathrm{cm}^{-1}$ for $n=4\left(620 \mathrm{~nm}\right.$ exc.) perovskite crystals. ${ }^{2}$ The initial photogenerated total

carrier density $[\mathrm{TC}]_{0}$ is calculated by the equation of $[\mathrm{TC}]_{0}=\alpha \frac{E \lambda}{h c \times \pi r^{2} N_{\text {layer }}}\left(1-\mathrm{e}^{-\varepsilon d}\right.$ )( $\left.1-R_{\text {pump }}\right)$, where the $\alpha$ is the ratio of charge carrier created by each photon absorbed, which is assumed to be $1 ; E$ is the energy of a single excitation pulse at wavelength $\lambda ; h$ is the Planck's constant, $c$ is the speed of light; $\varepsilon$ is the absorption 
coefficient; $d$ is the optical depth $(d=1 / \varepsilon, 267 \mathrm{~nm}$ for $n=2,323 \mathrm{~nm}$ for $n=3$ and 364 $\mathrm{nm}$ for $n=4)$; $R_{\text {pump }}$ is the reflectivity of cover glass at normal incidence of the excitation beam $\left(R_{\text {pump }} \approx 0\right) ; r$ is the radius of excitation spot $(r=150 \mu \mathrm{m})$; and $N_{\text {layer }}$ is the number of perovskite layers and can be determined by equation of $N_{\text {layer }}=$ $d / d_{\text {layer }}(136$ for $n=2,124$ for $n=3$ and 113 for $n=4)$, where $d_{\text {layer }}$ is the thickness of the mono perovskite layers $(1.97 \mathrm{~nm}$ for $n=2,2.60 \mathrm{~nm}$ for $n=3$ and $3.22 \mathrm{~nm}$ for $n=$ $4) .^{3}$

\section{References}

(1) Lai, R.; Wu, K. Picosecond electron trapping limits the emissivity of $\mathrm{CsPbCl}_{3}$ perovskite nanocrystals. J. Chem. Phys. 2019, 151, 194701.

(2) Deng, S.; Shi, E.; Yuan, L.; Jin, L.; Dou, L.; Huang, L. Long-range exciton transport and slow annihilation in two-dimensional hybrid perovskites. Nat. Commun. 2020, 11, 664.

(3) Cao, D. H.; Stoumpos, C. C.; Farha, O. K.; Hupp, J. T.; Kanatzidis, M. G. 2D Homologous Perovskites as Light-Absorbing Materials for Solar Cell Applications. J. Am. Chem. Soc. 2015, 137, 7843-7850. 
Table S1. Experimental and simulated (according to Saha equation and steady-state $\left.E_{\mathrm{b}}\right)$ equilibrated exciton-to-free-carrier ratio $([\mathrm{X}] /[\mathrm{FC}])$ for $2 \mathrm{D}$ perovskites with $n=2$, 3 and 4, where the densities of total carrier are 4.0, 1.32 and $2.53 \times 10^{11} \mathrm{~cm}^{-2}$ layer $^{-1}$, respectively.

\begin{tabular}{cccc}
\hline & $\boldsymbol{n}=\mathbf{2}$ & $\boldsymbol{n}=\mathbf{3}$ & $\boldsymbol{n}=\mathbf{4}$ \\
\hline Experimental & 0.24 & 0.16 & 0.16 \\
Simulated & 15.7 & 3.04 & 2.85 \\
\hline
\end{tabular}

Table S2. Temperature-dependent exciton dissociation time $\tau_{\text {dis. }}$ (ps) of $n=2-4$ perovskite QWs, determined by exponential fit of PL decays measured under various temperatures (in Figure 1e and S8).

\begin{tabular}{cccc}
\hline Temperature (K) & $\boldsymbol{n}=\mathbf{2}$ & $\boldsymbol{n}=\mathbf{3}$ & $\boldsymbol{n}=\mathbf{4}$ \\
\hline $\mathbf{8 5}$ & 16.78 & 5.96 & 2.13 \\
$\mathbf{1 5 5}$ & 9.30 & 2.26 & 1.81 \\
$\mathbf{2 2 5}$ & 3.16 & 1.34 & 1.20 \\
$\mathbf{3 6 0}$ & 2.27 & 0.94 & 0.57 \\
\hline
\end{tabular}


Table S3. The exciton binding energy $\left(E_{\mathrm{b}}\right)$ of $(\mathrm{PEA})_{2}(\mathrm{MA})_{\mathrm{n}-1} \mathrm{~Pb}_{\mathrm{n}} \mathrm{I}_{3 \mathrm{n}+1}(n=1-4) 2 \mathrm{D}$ perovskites from reported experimental results $(n=1$ and 2, Ref 37 in maintext) and calculated according to the scaling law proposed by Blancon et al. ( $n=3$ and 4 , Figure S14).

\begin{tabular}{cc}
\hline $\boldsymbol{n}$ & $\boldsymbol{E}_{\mathrm{b}} / \mathbf{m e V}$ \\
\hline $\mathbf{1}$ & 220 \\
$\mathbf{2}$ & 170 \\
$\mathbf{3}$ & 123 \\
$\mathbf{4}$ & 99 \\
\hline
\end{tabular}

Table S4. The exponential fitting parameters for long-range PL decay kinetics of 2D perovskite with $n=2-4$ (Figure $\mathrm{S} 10)$, where $\tau_{\mathrm{x}}$ and $A_{\mathrm{x}}(\mathrm{x}=1,2)$ are the fitting lifetimes (ns) and the corresponding amplitudes.

\begin{tabular}{ccc}
\hline $\boldsymbol{n}$ & $\tau_{\mathbf{1}}\left(\boldsymbol{A}_{\mathbf{1}}\right) / \mathbf{n s}$ & $\boldsymbol{\tau}_{\mathbf{2}}\left(\boldsymbol{A}_{\mathbf{2}}\right) / \mathbf{n s}$ \\
\hline $\mathbf{2}$ & $5.16(0.96)$ & $32.67(0.04)$ \\
$\mathbf{3}$ & $2.48(0.96)$ & $49.03(0.04)$ \\
$\mathbf{4}$ & $7.19(0.81)$ & $68.55(0.19)$ \\
\hline
\end{tabular}



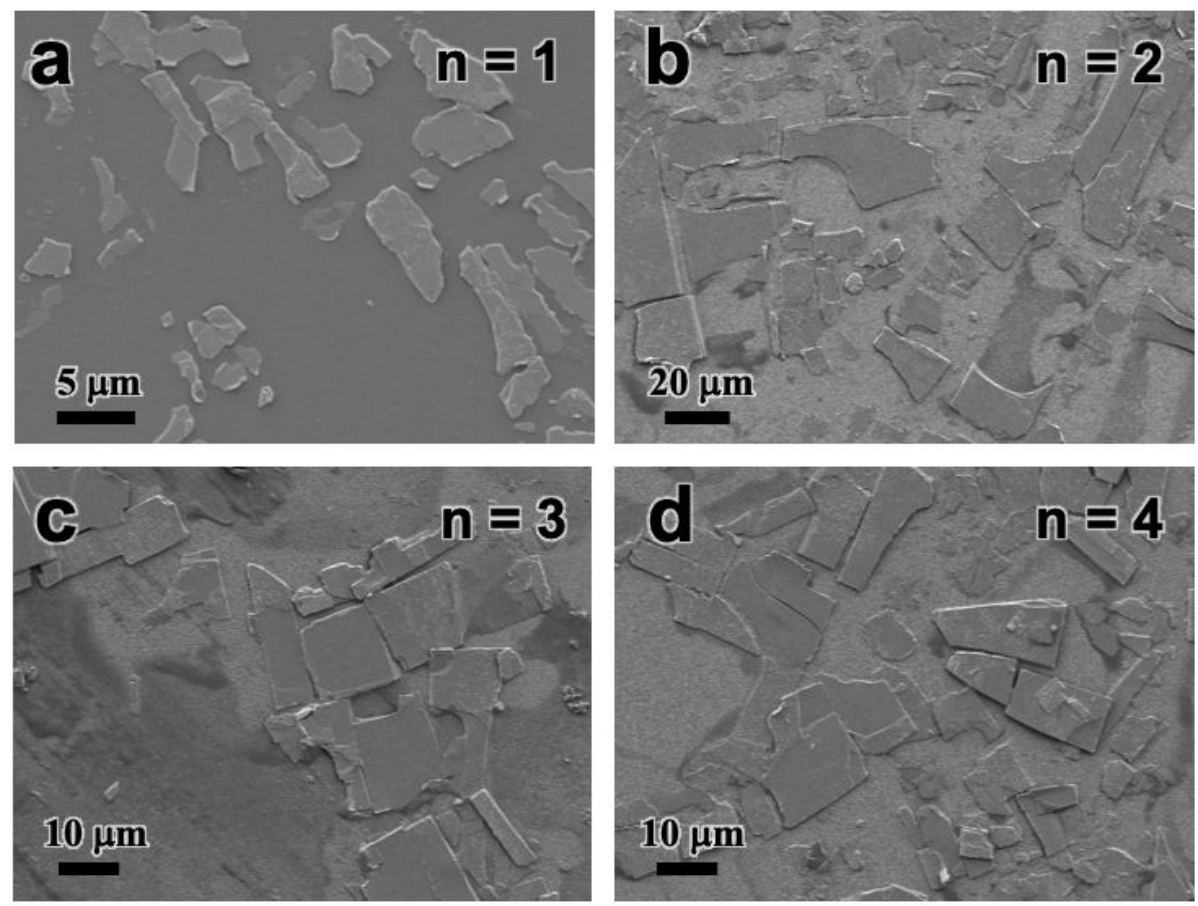

Figure S1. SEM images of mechanically exfoliated 2D perovskite SCs with $n=1-4$. 


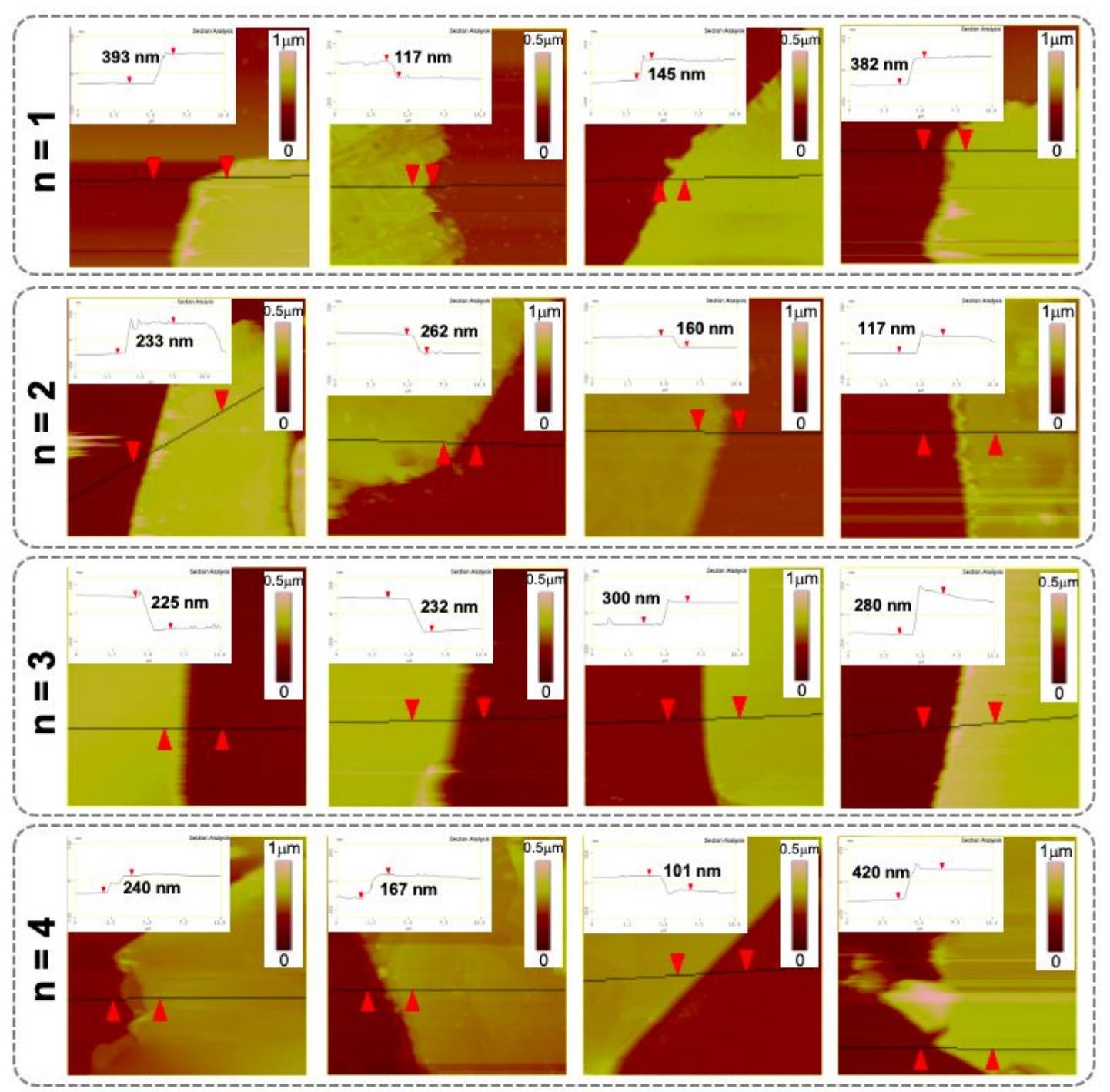

Figure S2. AFM images and one-dimensional height profiles (insets) revealing that the thickness of exfoliated SCs is about $100-400 \mathrm{~nm}$. Scan areas are $10 \mu \mathrm{m} \times 10 \mu \mathrm{m}$. 

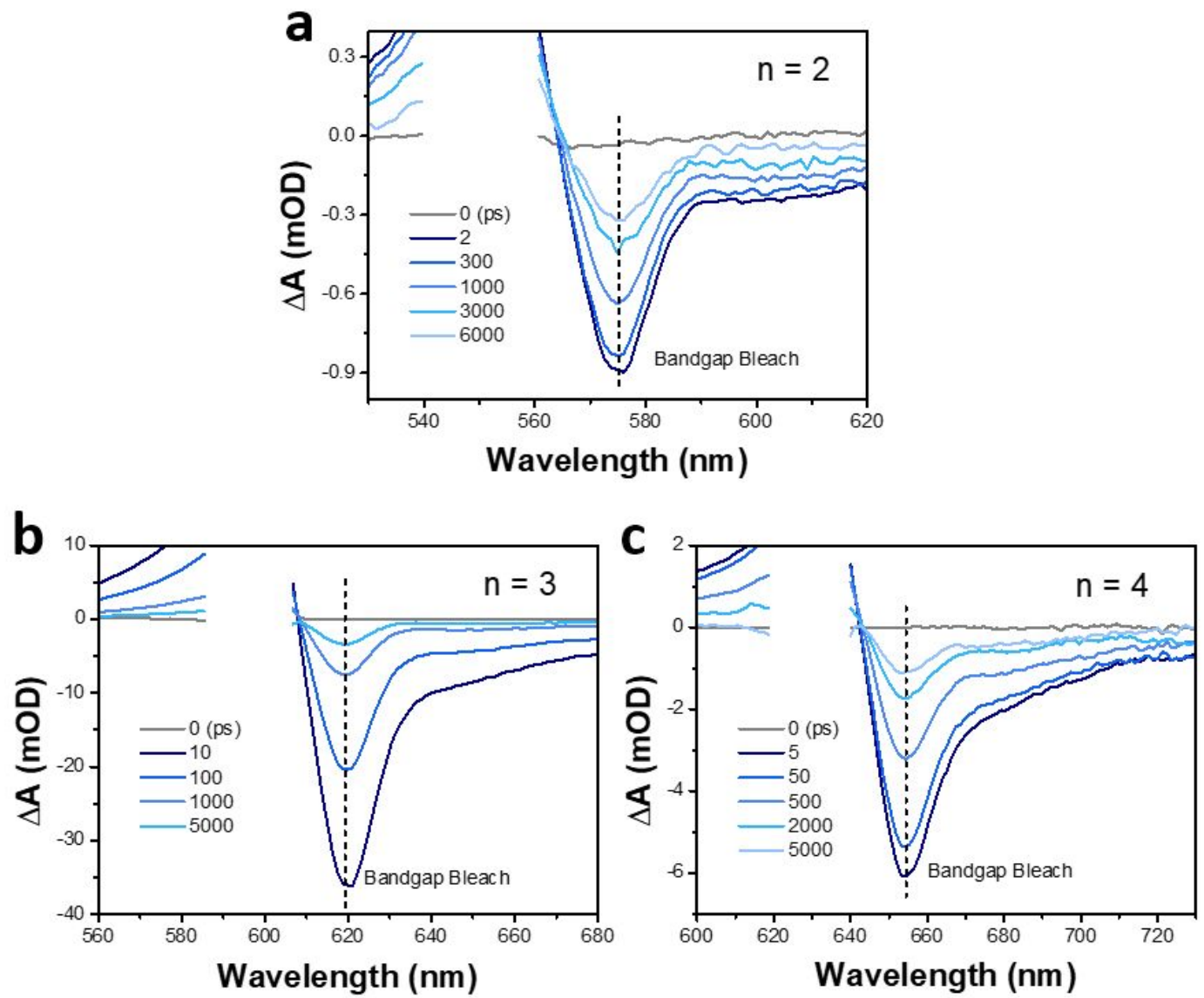

Figure S3. TA spectra of 2D perovskite QWs $(n=2-4)$ at indicated delay times with excitation wavelengths of 550, 600 and $620 \mathrm{~nm}$, respectively. 

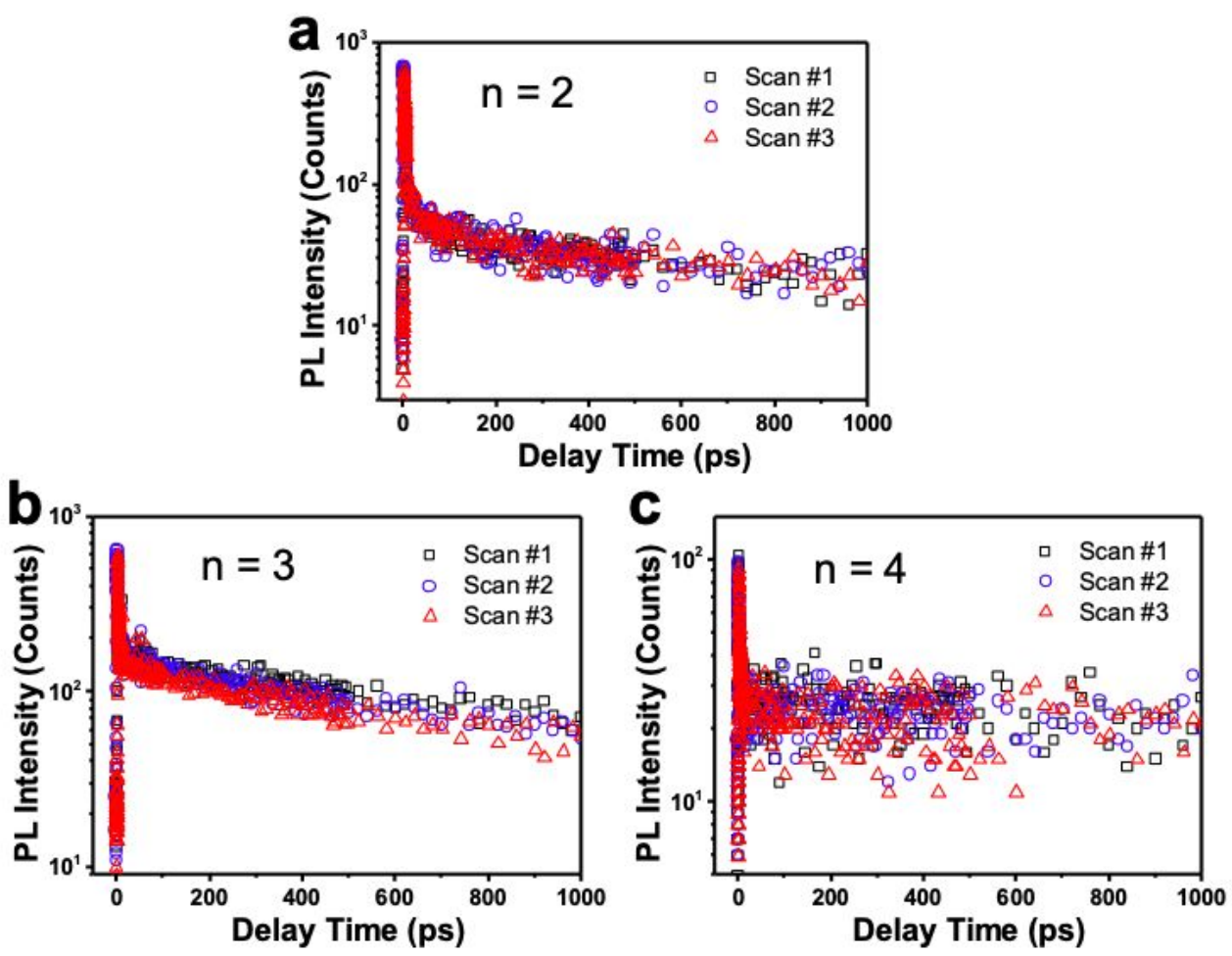

Figure S4. PL-UC kinetics collected at different experiment scans (within a time period of $1.5 \mathrm{~h}$ ) are almost identical, confirming that the exfoliated SCs are photo-stable during ultrafast spectroscopic measurements. 

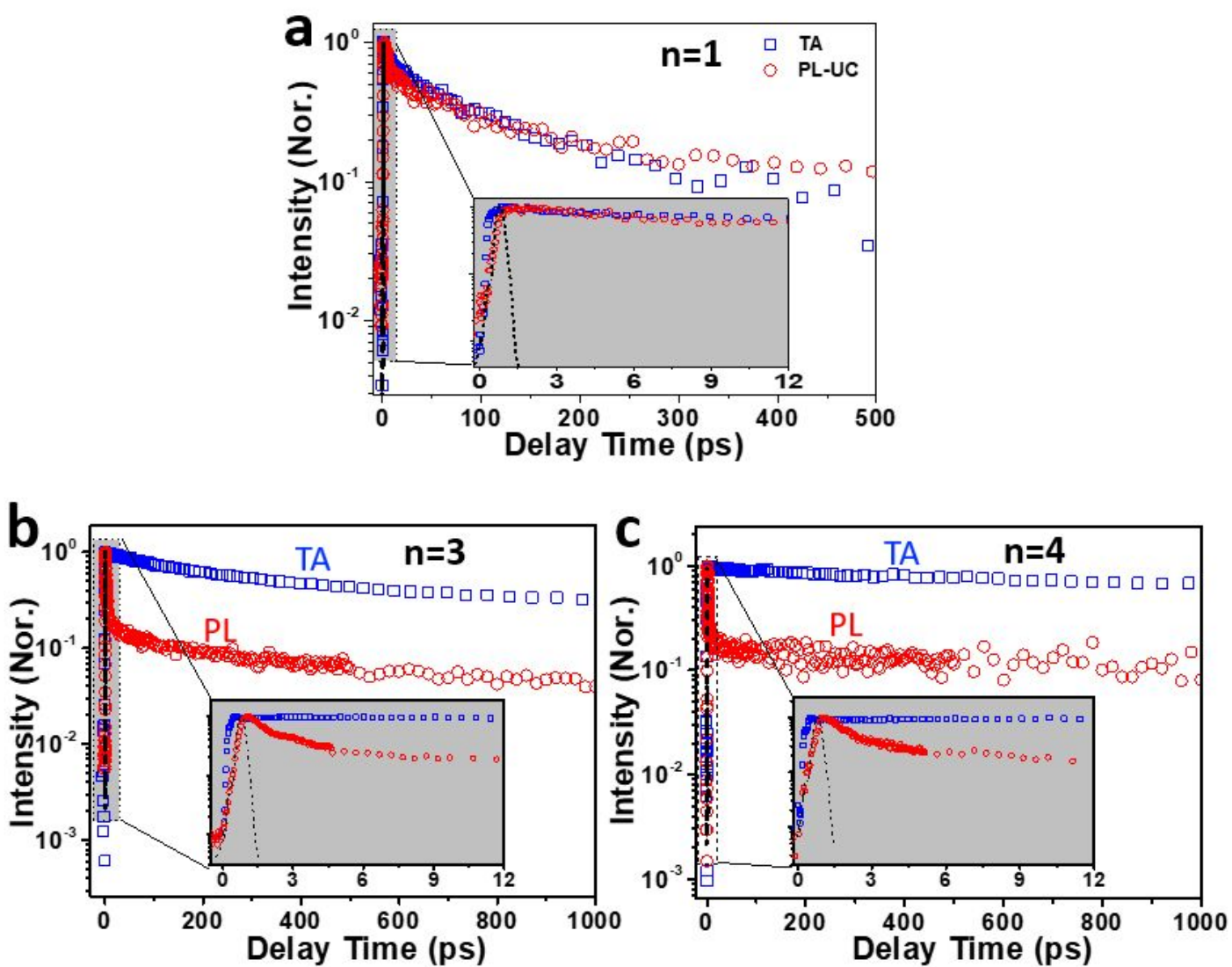

Figure S5. Comparison of TA and PL-UC kinetics of $n=1,3$ and 4 perovskite QWs under close bandgap excitation (500, 600 and $620 \mathrm{~nm}$, respectively). Insets are zoomed-in views for the first 12 ps delay and the dash lines are the instrument response function (IRF) of PL-UC setup. 

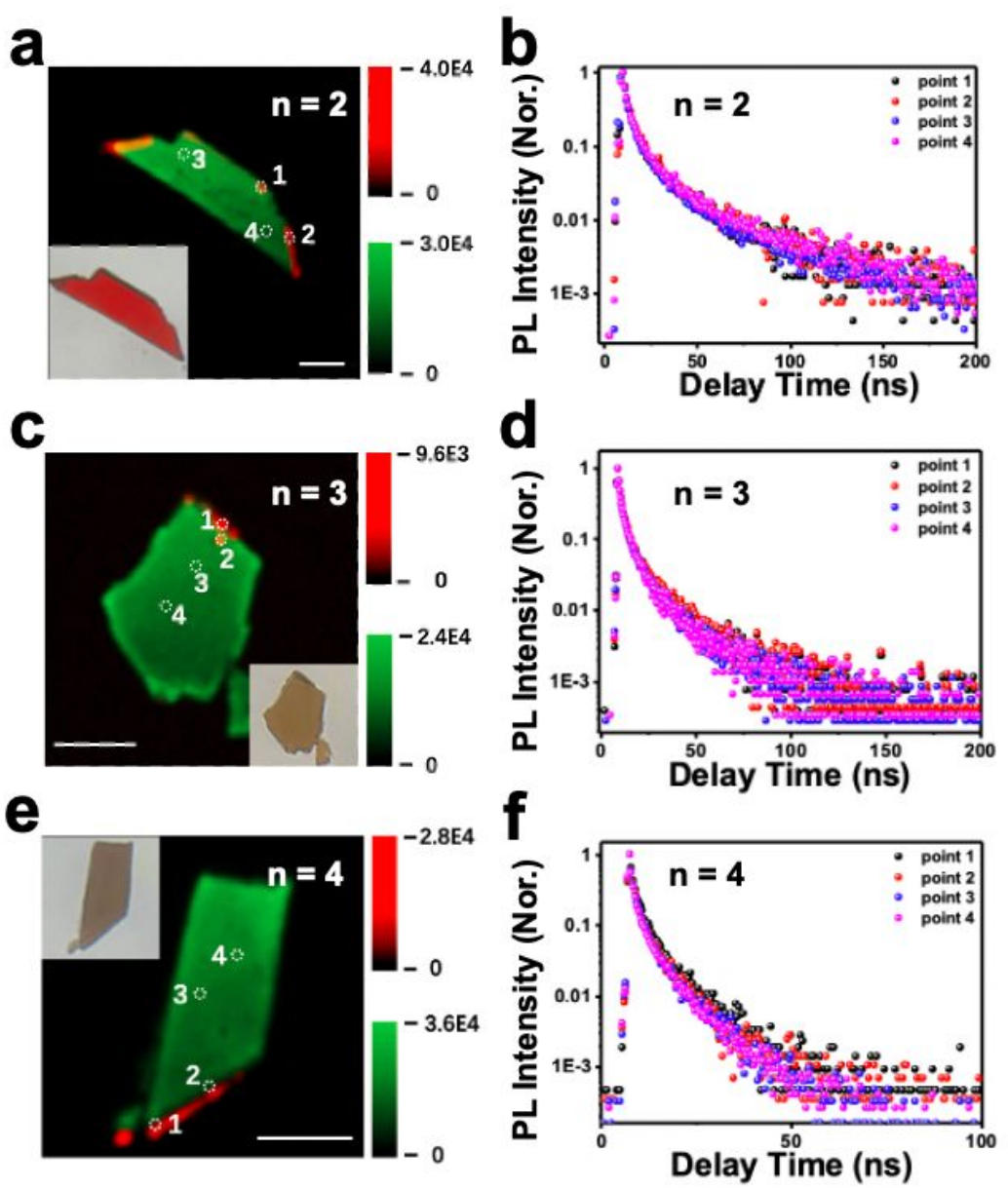

Figure S6. (a, c and e) PL intensity images of exfoliated 2D perovskite SCs $(n=2,3$ and 4, respectively) comprised of both exciton (green) and "edge-state" (red) emissions, obtained by a laser-scanned confocal imaging microscope coupled with time-correlated single photon counting $($ TCSPC $)\left(\lambda_{\text {exc. }}=405 \mathrm{~nm}\right)$. The insets show the optical images of SCs. Scale bars are $5 \mu \mathrm{m}$. (b, d and f) Exciton PL decays collected at different locations (as labeled in the left panels) with various distances to the edge-states show identical kinetics. This evidences that the presence of edge-state does not affect the intrinsic exciton kinetics in 2D perovskite. 

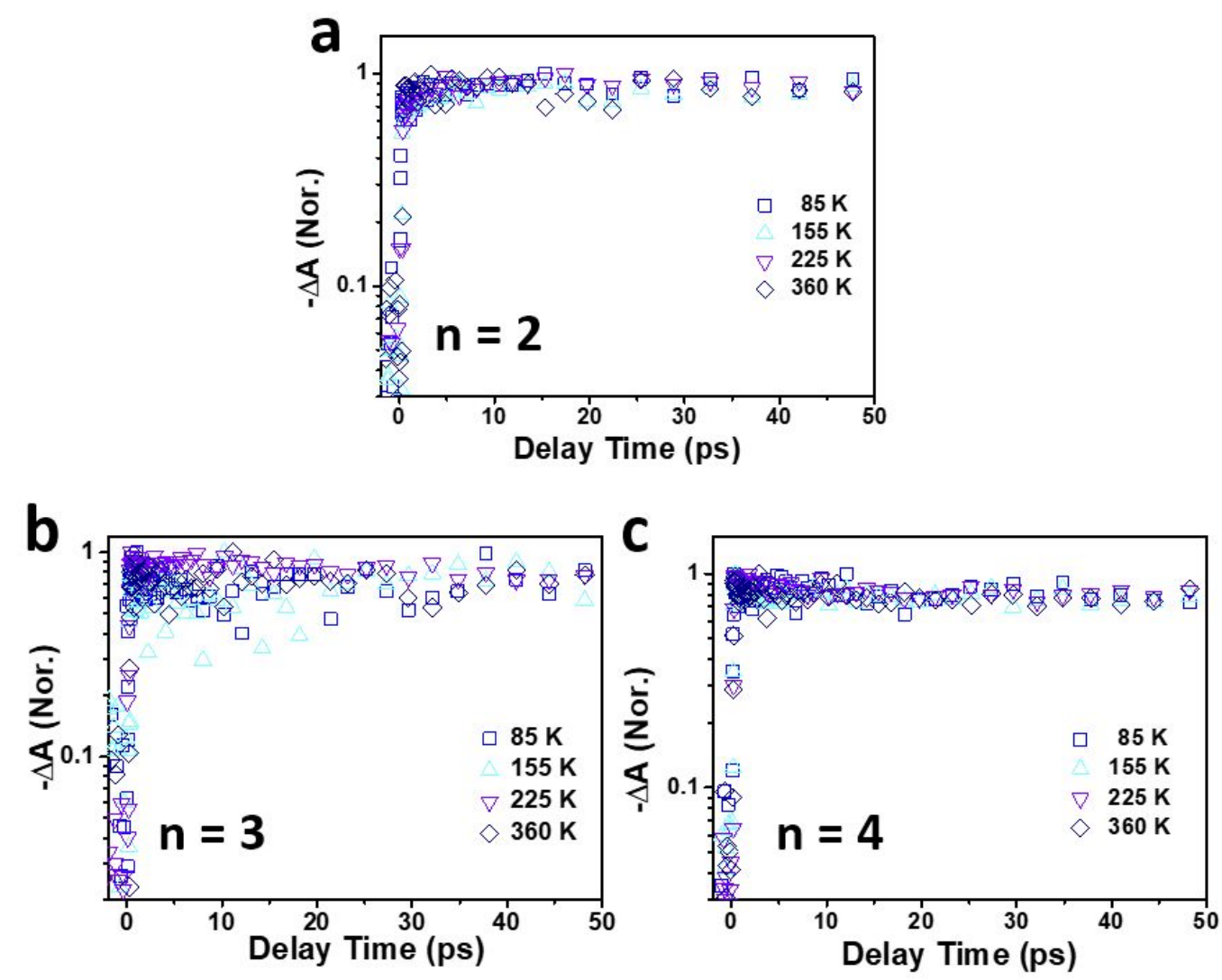

Figure S7. Temperature-dependent TA kinetics (monitored at bandgap bleach) of $n=$ 2-4 perovskite QWs under close-bandgap excitation, showing the almost identical TA recovery kinetics at different temperatures. 

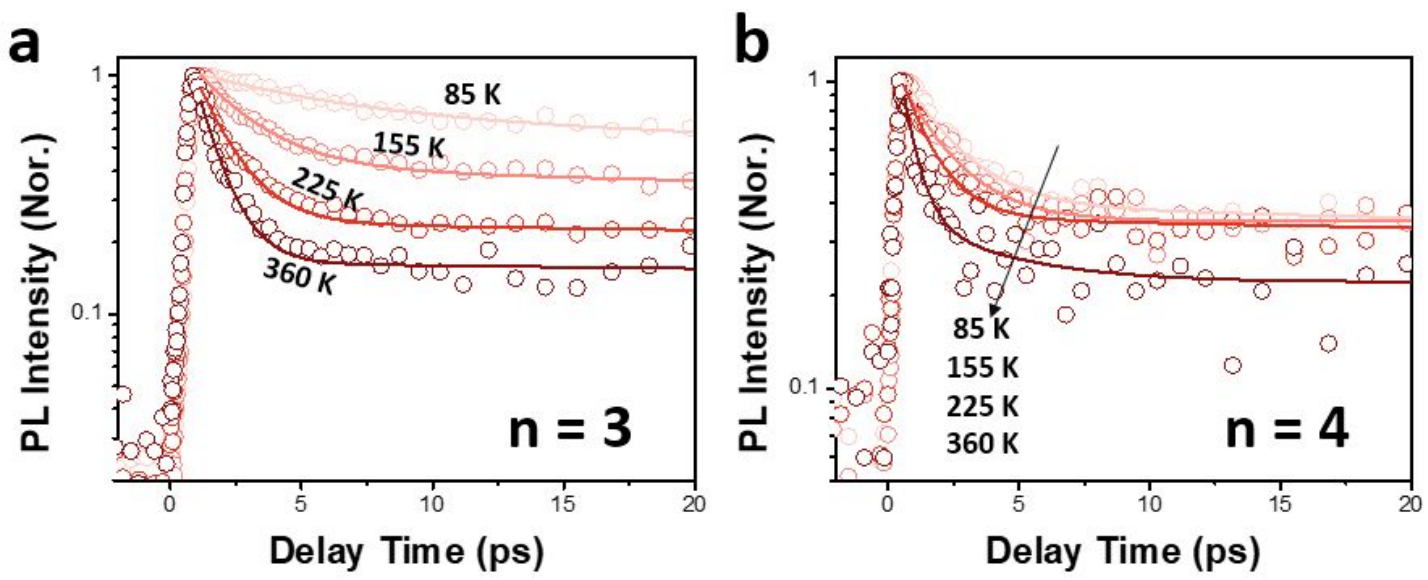

Figure S8. Temperature-dependent PL-UC decays of $n=3$ and 4 perovskite QWs under close-bandgap excitation. The solid lines are exponential fits. 

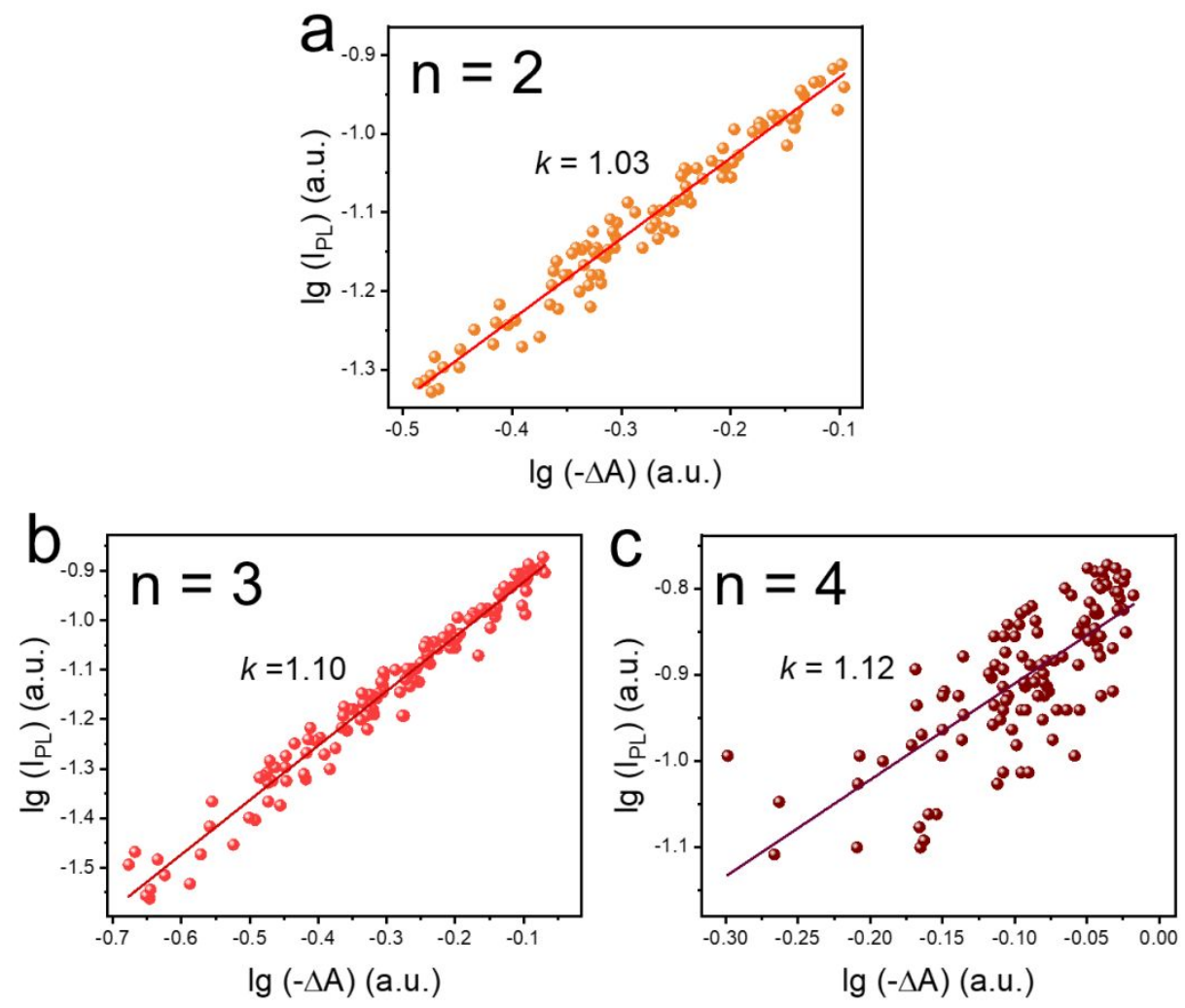

Figure S9. The plots of $I_{\mathrm{PL}} v s . \Delta \mathrm{A}$ in logarithmic scales following the law of $I_{\mathrm{PL}} \propto$ $\Delta \mathrm{A}^{\mathrm{k}}$, where the $k \approx 1$ indicating that the major luminescent carrier specie is exciton in $n=2-4$ perovskite QWs due to the first-order radiative recombination. 


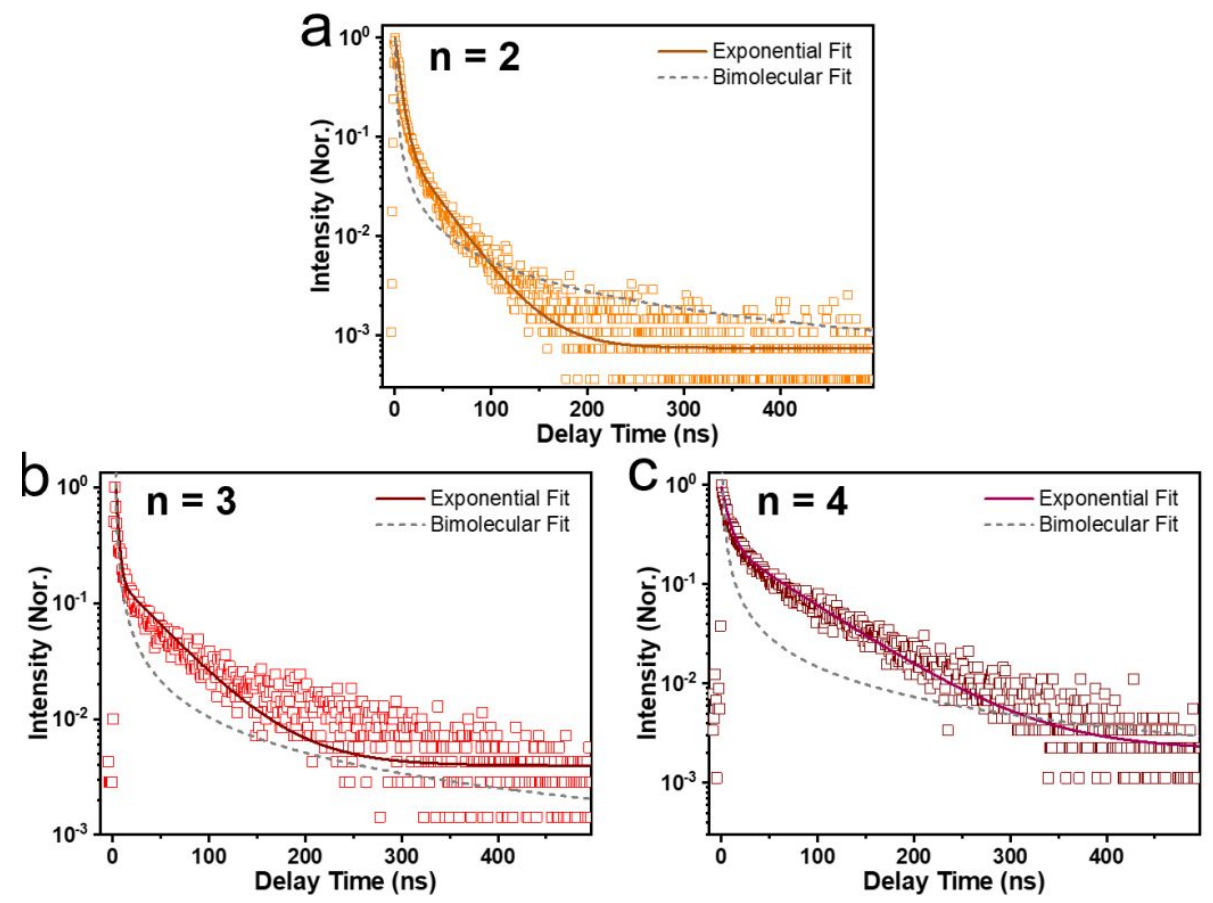

Figure S10. PL kinetics of $n=2-4$ perovskites collected within a time window of $500 \mathrm{~ns}$ by using TCSPC $\left(\lambda_{\mathrm{exc}}=405 \mathrm{~nm}\right)$, where the PL kinetics can be well fitted by an exponential function (solid lines, with fitting parameters listed in Table S4) but not bimolecular function (dash lines). This evidences that even though free-carries are dominant after dissociation equilibrium, the PL is still mainly from the first-order radiative recombination of exciton. 


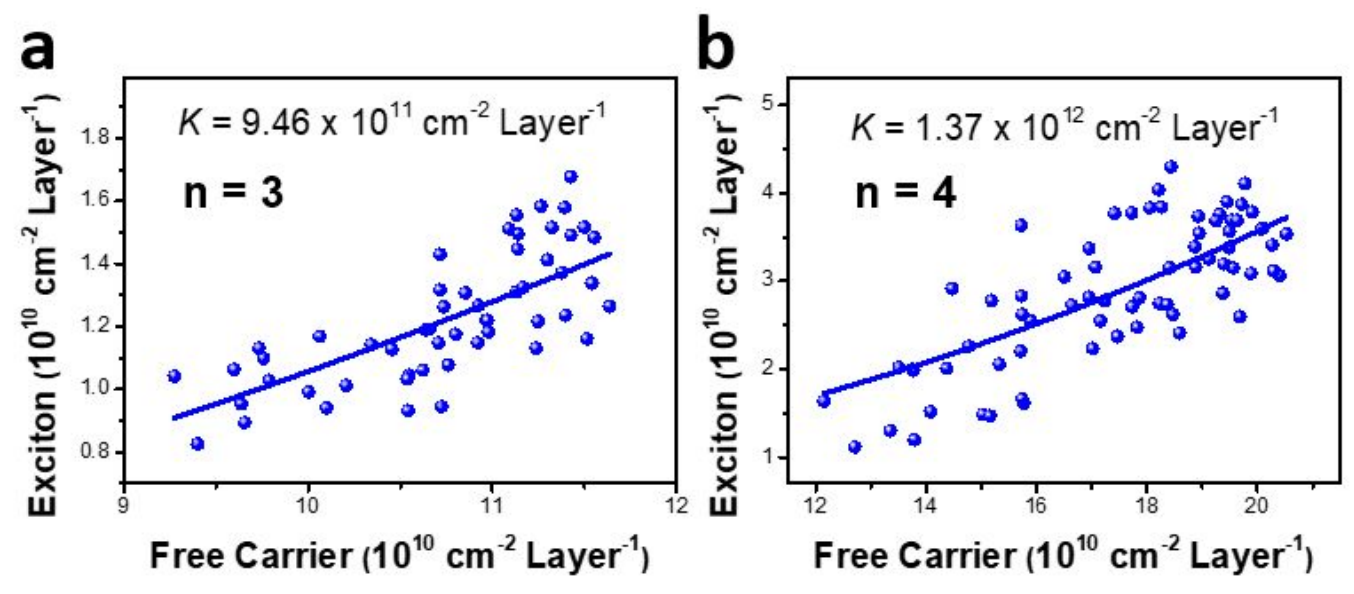

Figure S11. The exciton-free-carrier equilibrium constant $(K)$ of $n=3$ and 4 perovskite QWs fitted by the reversible reaction model of $K=k_{\mathrm{dis}} / k_{\mathrm{rb} .}=[\mathrm{FC}]^{2} /[\mathrm{X}]$. The solid lines are best fits. The data points in figures are obtained from the TA and PL-UC kinetics after the exciton dissociation reaching dynamical equilibrium (Figure $1 \mathrm{~d}$ and $\mathrm{S} 5)$. The $\Delta \mathrm{A}$ signal indicates the change of the total carrier density $[\mathrm{TC}](\mathrm{t})$ as a function of delay time: $\Delta \mathrm{A}(\mathrm{t}) \propto[\mathrm{TC}](\mathrm{t})=[\mathrm{FC}](\mathrm{t})+[\mathrm{X}](\mathrm{t})$. And in the PL-UC kinetics, the PL intensity is $I_{\mathrm{PL}}(\mathrm{t}) \propto[\mathrm{X}](\mathrm{t})$; this is because that the PL of $2 \mathrm{D}$ perovskites is mainly from the radiative recombination of excitons rather than free-carriers (Figure S9 and S10). Under the close-bandgap excitation, the initial carriers are excitons, and PL-UC and TA kinetics reflect the density ratio between $[\mathrm{FC}]$ and $[\mathrm{X}]$ at different delay times. Combining the initial total carrier density $[\mathrm{TC}]_{0}$ (see calculation details in Supplementary Note 4), a set of $[\mathrm{FC}]$ and $[\mathrm{X}]$ data points at equilibrium state can be obtained for $K$ fitting. 

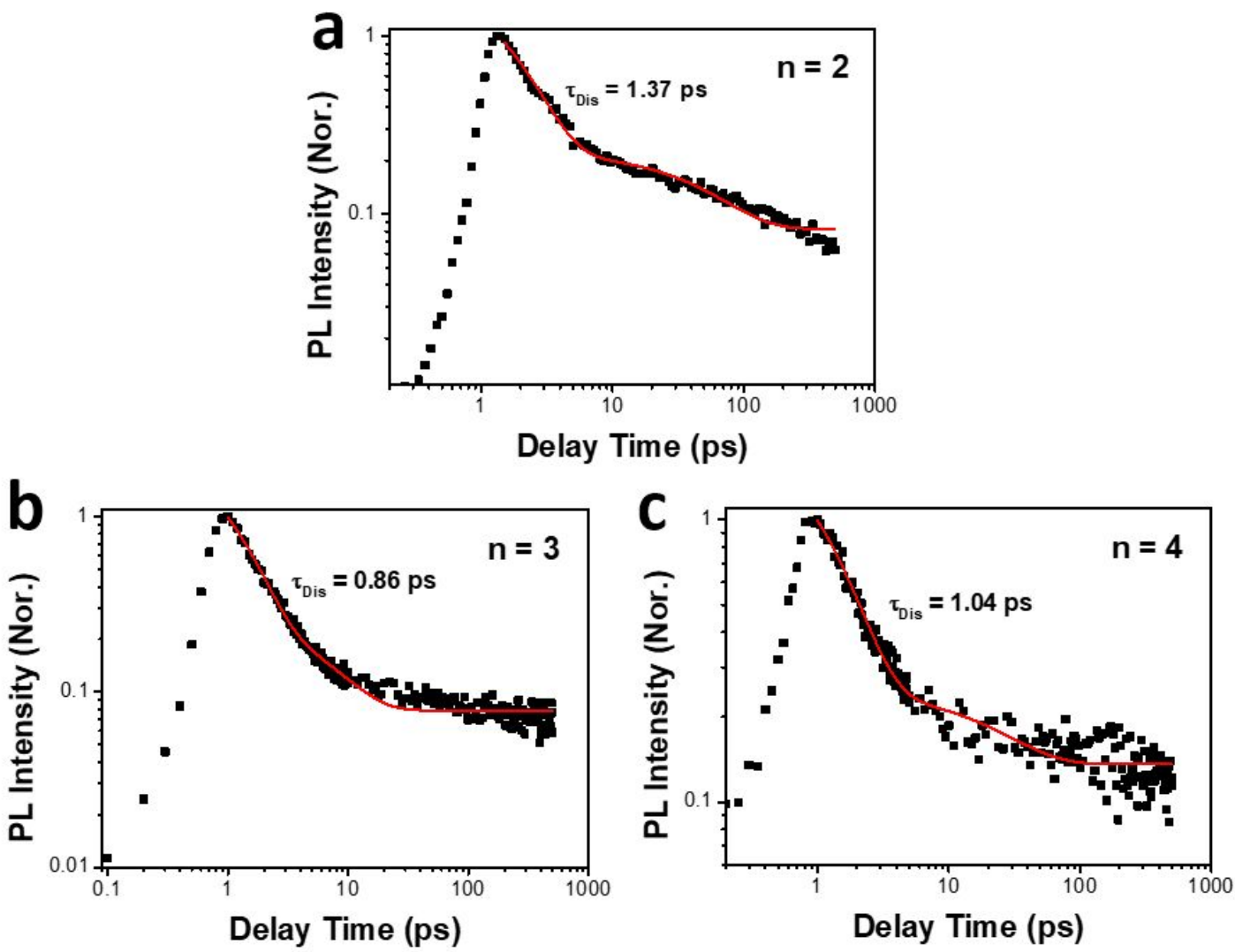

Figure S12. The dissociation rates $\left(k_{\text {dis. }}=1 / \tau_{\text {dis. }}\right)$ of $n=2-42 \mathrm{D}$ perovskites were determined by exponential fit (red solid lines) of PL-UC decays. 

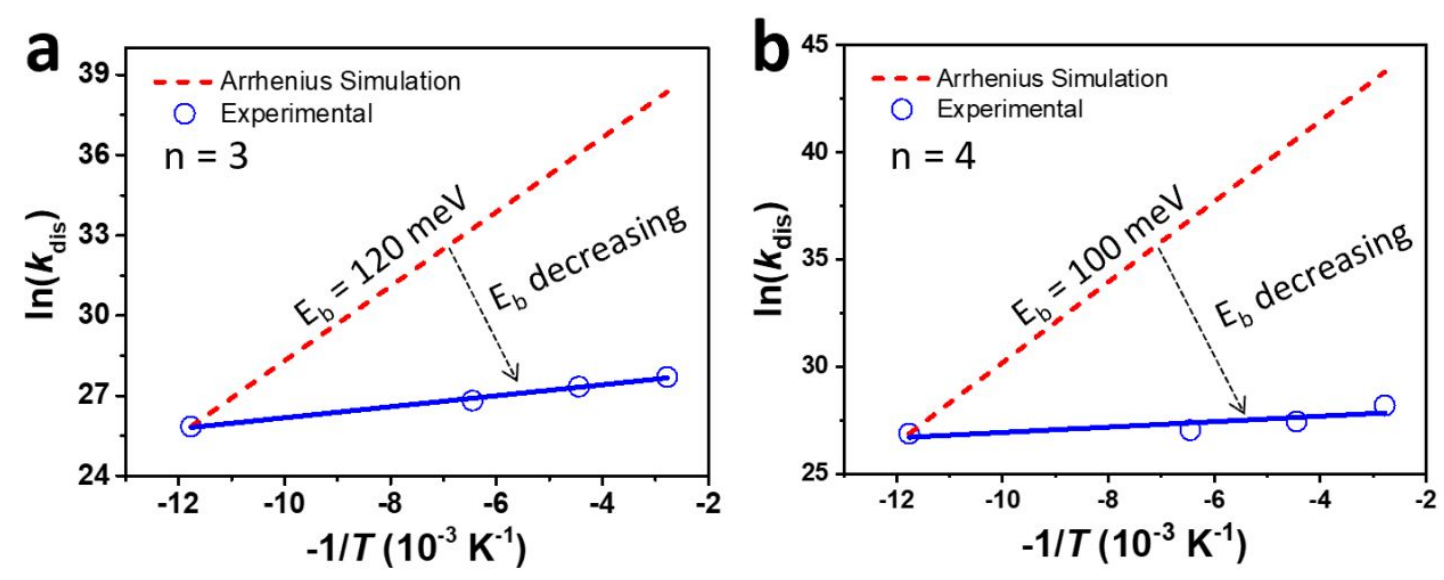

Figure S13. The Arrhenius plots of natural logarithm of $k_{\text {dis. }}\left(\ln \left(k_{\text {dis. }}\right)\right)$ as a function of negative reciprocal of temperature $(-1 / T)$ for $n=3$ (a) and 4 (b) 2D perovskites. The red dash lines show the simulated Arrhenius plot of $\ln \left(k_{d i s .}\right)$ vs. $(-1 / \mathrm{T})$ by using the steady state $E_{\mathrm{b}}$ (120 and $100 \mathrm{meV}$ for $n=3$ and 4, respectively). The large discrepancy between the simulated and experimental plots implies a decreasing exciton binding energy in the experimental measurement. 


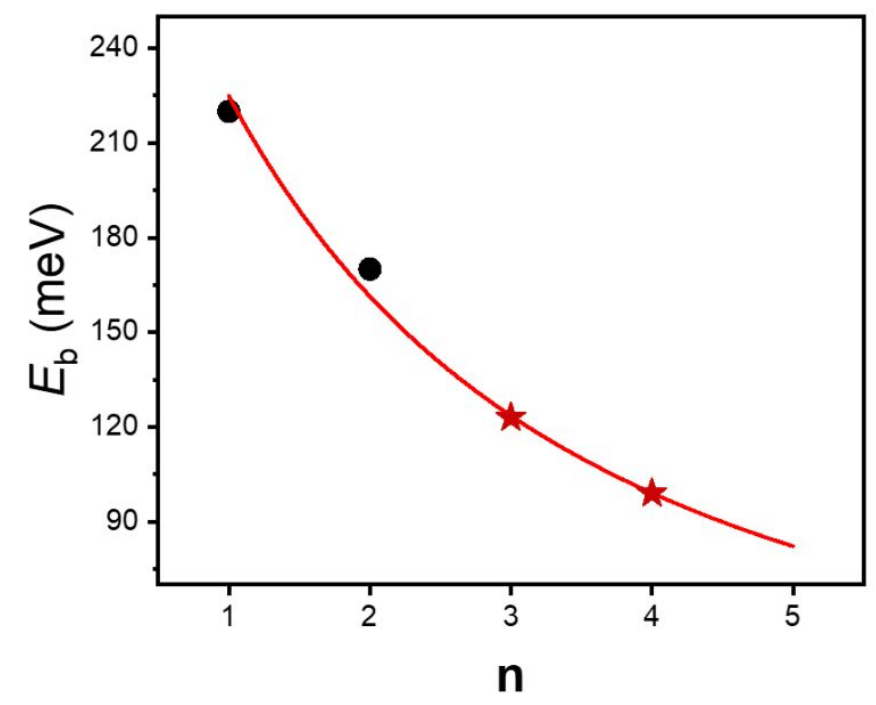

Figure S14. The exciton binding energy $\left(E_{\mathrm{b}}\right)$ of $n=3$ and 4 2D perovskites (labeled as stars) are estimated from the reported values of $n=1,2$ (Ref. 37 in main text) and by using the scaling law proposed by Blancon et al. (solid line, Ref. 15 in main text). 

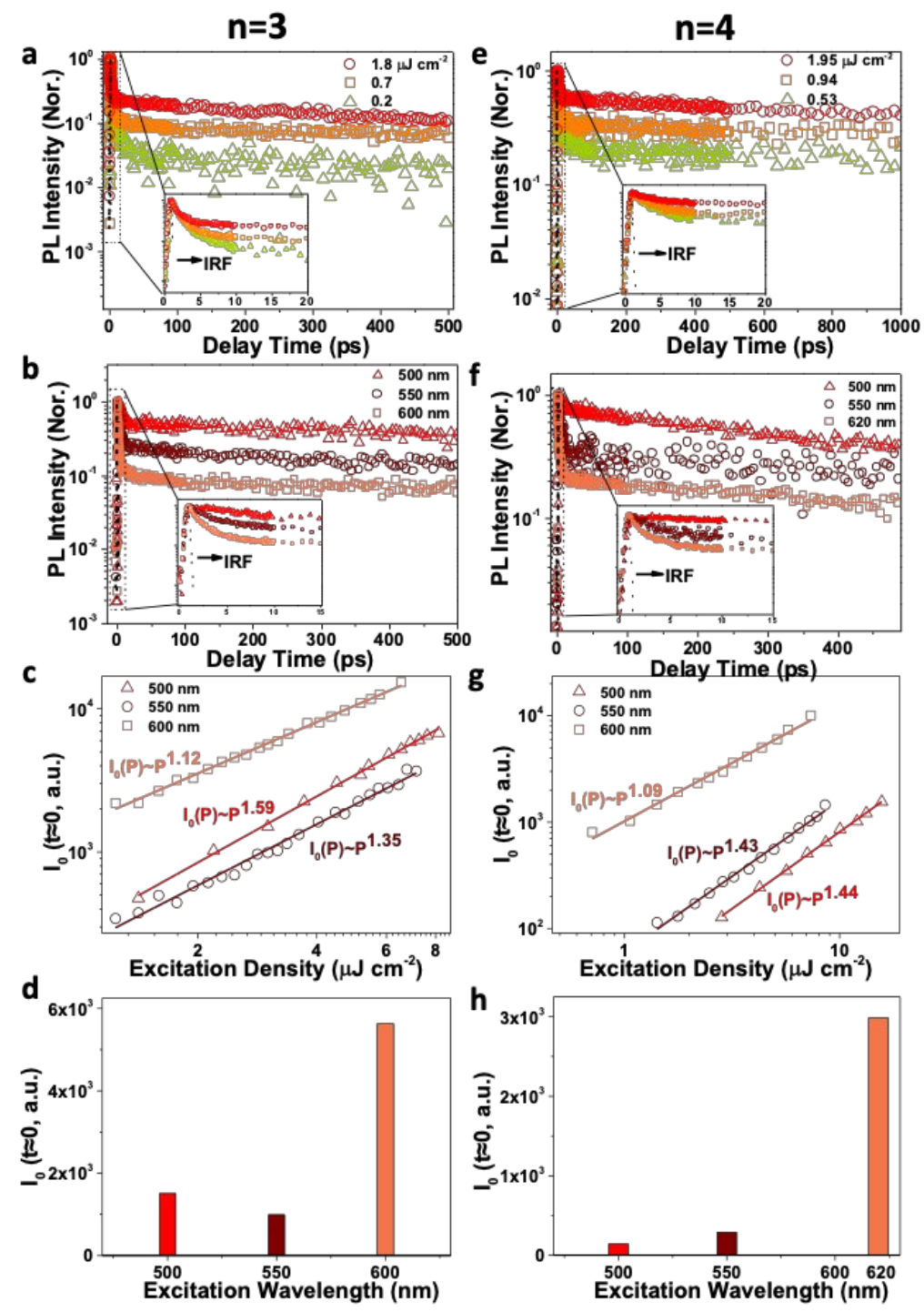

Figure S15. Excitation-intensity- and excitation-wavelength-dependent PL-UC measurements of $n=3$ (left panels) and 4 (right panels) 2D perovskite QWs. Panels $(a, e)$ and $(b, f)$ show excitation-intensity- and wavelength-dependent PL kinetics, respectively. Insets are zoomed-in views of the kinetics in the first tens of ps. (c, g) The initial maximum PL intensities $\left(I_{0}\right.$, at delay time $\left.\mathrm{t} \approx 0 \mathrm{ps}\right)$ as a function of excitation intensity measured under different excitation wavelengths for $n=3$ and 4 perovskite QWs, which follows the law of $I_{0}(P) \sim P^{\alpha}$ (solid lines). (d, h) Comparisons of $I_{0}$ under different excitation wavelength with the same total carrier densities in $n=$ 3 and 4 perovskites. 

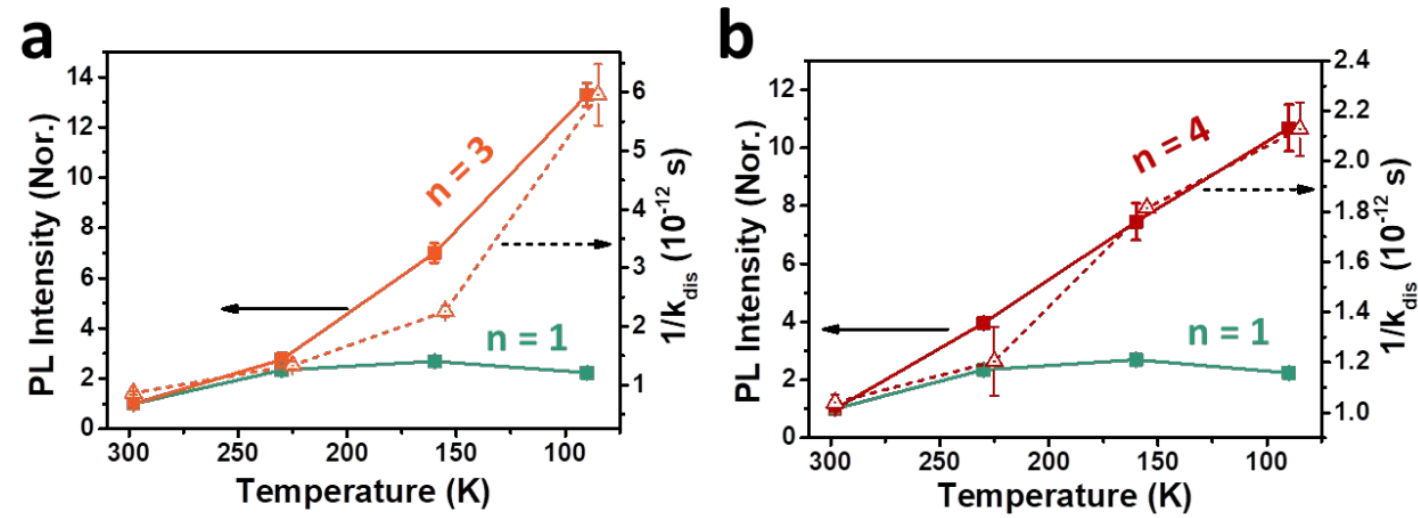

Figure S16. Temperature-dependent PL intensity collected from $n=3$ (a) and 4 (b)

2D perovskites. The reciprocals of dissociation rate $\left(1 / k_{\text {dis. }}\right.$, hallow triangles and dash lines) exhibit a similar trend with PL intensity. 